\title{
Correction coefficient for see-through labyrinth seal
}

\author{
Dan Hasnedl ${ }^{1, *}$, Premysl Epikaridis ${ }^{1}$, and Vaclav Slama ${ }^{1}$ \\ ${ }^{1}$ Doosan Skoda Power, R\&D Department, Tylova 1/57, Pilsen, Czech republic
}

\begin{abstract}
In a steam turbine design, the flow-part design and blade shapes are influenced by the design mass-flow through each turbine stage. If it would be possible to predict this mass-flow more precisely, it will result in optimized design and therefore an efficiency benefit. This article is concerned with improving the prediction of losses caused by the seal leakage. In the common simulation of the thermodynamic cycle of a steam turbine, analytical formulas are used in order to simulate the seal leakage. Therefore, this article describes an improvement of analytical formulas used in a turbine heat balance calculation. The results are verified by numerical simulations and experimental data from the steam test rig.
\end{abstract}

\section{Nomenclature}

m mass flow

S flow through area

$\mathrm{p}_{0} \quad$ inlet absolute pressure

$\mathrm{p}_{2} \quad$ outlet absolute pressure

$\left(\mathrm{p}_{2} / \mathrm{p}_{0}\right)_{\text {cr }}$ critical pressure ratio

$\mathrm{p}_{\mathrm{i}} \quad$ absolute pressure after the tooth " $i$ "

$\mathrm{p}_{\mathrm{n}} \quad$ absolute pressure after the last tooth

$\mathrm{v}_{0} \quad$ inlet specific volume

$\kappa \quad$ Poisson's constant

$\delta$ radial clearance

$\mathrm{s} \quad$ span of the teeth

$\mathrm{z} \quad$ number of the teeth

$\mathrm{k} \quad$ see-through seal correction coefficient

$\mu \quad$ flow contraction correction coefficient

\section{Introduction}

Classical labyrinth seals are still widely used due to their cost, simple and robust design despite of new types of seals, such as brush seals. In the turbine heat balance or efficiency calculations, an estimation of the leakage through these seals is represented by analytical formulas , which can be used to carry out calculations fast enough in order to save time especially during the bidding procedure. For these purposes, the time consuming numerical calculations are not feasible.

Several authors managed to estimate the labyrinth seal leakage by various formulas, such as Zalf [5], Kearton [6], Samoylovich [4] and others [1], [2], [7], [8]. These formulas are different in an approach and results. Over a number of years, Zalf formula was used due to its simplicity and conservative results in Doosan Skoda leakage predictions. In a modern turbine design, where a high efficiency is demanded, it is crucial to use more enhanced and precise way to estimate the leakage.
Therefore, a large set of experiments and numerical calculations were performed to compare the results. Stepped labyrinth and see-through labyrinth seals with different clearances were tested in steam environment to get as close to the real operating conditions as possible.

All geometric combinations were also simulated by CFD software (FLUENT, SEAL) as the alternative sources of leakage values.

\section{Analytical formulas}

As a representative of the common practice, the Zalf formula was brought for comparison. Zalf formula takes into consideration only pressure ratio and number of the teeth. The leakage is estimated as:

$m=1.5 \cdot S \cdot \sqrt{\frac{p_{0}}{v_{0}}} \cdot \sqrt{\kappa \cdot\left(\frac{2}{\kappa+1}\right)^{\frac{\kappa+1}{\kappa-1}}} \cdot \sqrt{\frac{1-\left(p_{2} / p_{0}\right)^{2}}{z-\ln \left(p_{2} / p_{0}\right)}}$

The see-through labyrinth is simulated by using half number of the teeth (rounded) as long as the teeth span is lower than $16 \mathrm{~mm}$. For larger teeth span, the full number of the teeth is used. This rule comes from the technical practice where either much smaller span is used in combination with a high number of teeth or a large span is used in combination with a low number of the teeth.

A better correlation with experimental data is expected from Samoylovich formula, which keeps a reasonable level of simplicity despite consideration of pressure ratio, number, thickness, span and shape of the teeth.

$$
\begin{aligned}
& m=k \cdot \mu \cdot S \cdot \sqrt{\frac{p_{0}}{v_{0}}} \cdot \sqrt{\kappa \cdot\left(\frac{2}{\kappa+1}\right)^{\frac{\kappa+1}{\kappa-1}}} \\
& \cdot \sqrt{\frac{1-\left(p_{2} / p_{0}\right)^{2}}{z \cdot\left(1-\left(p_{2} / p_{0}\right)_{c r}\right)}-\frac{\left(p_{2} / p_{0}\right)_{c r} \cdot\left(1-p_{2} / p_{0}\right)^{2}}{\left(1-\left(p_{2} / p_{0}\right)_{c r}\right)^{2} \cdot z^{2}}}
\end{aligned}
$$

\footnotetext{
Corresponding author: dan.hasnedl@,doosan.com
} 
The see-through labyrinth is represented by a correction coefficient $k$, which is a function of the number of teeth, radial clearance and teeth span.

Added to this, the Kearton formula was brought for comparison despite its complexity, because it gives results in each inter-teeth chamber that can be later utilized for the seal mechanical design. The formula is:

$$
m=k \cdot \mu \cdot S \cdot \sqrt{\frac{p_{0}}{v_{0}}} \cdot \sqrt{F \cdot \frac{1-\left(p_{2} / p_{0}\right)^{2}}{z}}
$$

where

$$
F=\frac{\sum_{i=1}^{z} \varepsilon_{i}}{z}
$$

where

$$
\begin{aligned}
& \varepsilon_{i}=\frac{p_{i}}{p_{i-1}} \\
& p_{i}=\sqrt{p_{0}^{2}-\frac{i}{z} \cdot\left(p_{0}^{2}-p_{n}^{2}\right)}
\end{aligned}
$$

\subsection{Correction coefficients}

Coefficients $k, \mu$ are used to correct the leakage due to flow behaviour.

Flow contraction is represented by the correction coefficient $\mu$ that comes from the interpolation of the Sceglajev figure.

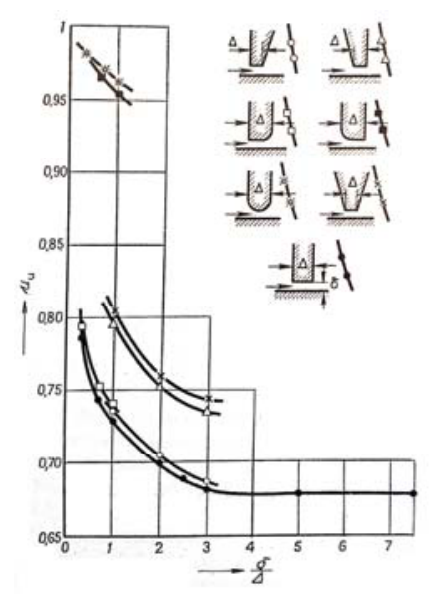

Fig. 1. Sceglajev figure for the evaluation of flow contraction correction coefficient

For calculation of the correction coefficient $k$, which takes in consideration flow in the see-through labyrinth seal, it was used the analytical formula in the first step:

$$
k=1+16 \cdot\left(1-e^{-0,1234 \cdot z}\right) \cdot \frac{\delta}{S}
$$

However, the experimental results didn't correlate with analytical approach (especially in cases where ratio $\delta / \mathrm{s}$ is more than 0.1 ). Therefore, a new analytical formula for correction coefficient $k$ has been designed:

$$
k=1+3,27 \cdot\left(1-e^{-0,4 \cdot z}\right) \cdot\left(\frac{\delta}{s}\right)^{\frac{2}{3}}
$$

\section{Experiments}

Experiments were carried out on the steam test rig which is situated in the R\&D laboratory of Doosan Skoda Power.

\subsection{Measured seal geometry}

Examples of typical stepped and see-through labyrinth seals are shown below. Figure 2 shows a typical stepped labyrinth while Figure 3 shows a seethrough design. The seals were tested with radial clearances varying from $0.2 \mathrm{~mm}$ to $0.9 \mathrm{~mm}$ and number of teeth from 2 to 6 .

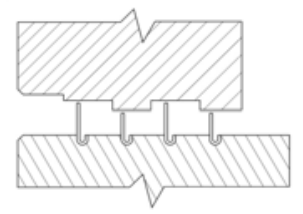

Fig. 2. Typical stepped labyrinth seal

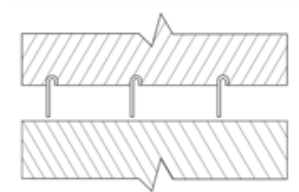

Fig. 3. Typical see-through labyrinth seal

\subsection{Steam test rig}

The steam test rig (shown on Figure 4) is used for a mass flow evaluation by measuring of the condensate volume (representing steam leakage through a seal). All steam, which passed through a seal, goes into an atmospheric condenser. From there, the steam condensate is collected in the tank, where the water level is measured. Calibrated volume between two water levels in combination with the time from the bottom to upper level gives the mass flow. The equipment for measuring the amount of the condensate is shown on Figure 5.

The test rig medium is a medium pressure steam supplied by the next-door power-plant at maximum parameters of $18 \mathrm{bar}_{\mathrm{g}}$ and $350^{\circ} \mathrm{C}$. Nevertheless, typical operating conditions are usually lower.

The test rig itself consists of a stationary casing and a rotating overhung shaft. The test section consists of an exchangeable stationary part as well as a rotating part. Therefore, various geometries can be tested. The seal diameter is (depending on the seal type) around $290 \mathrm{~mm}$. 


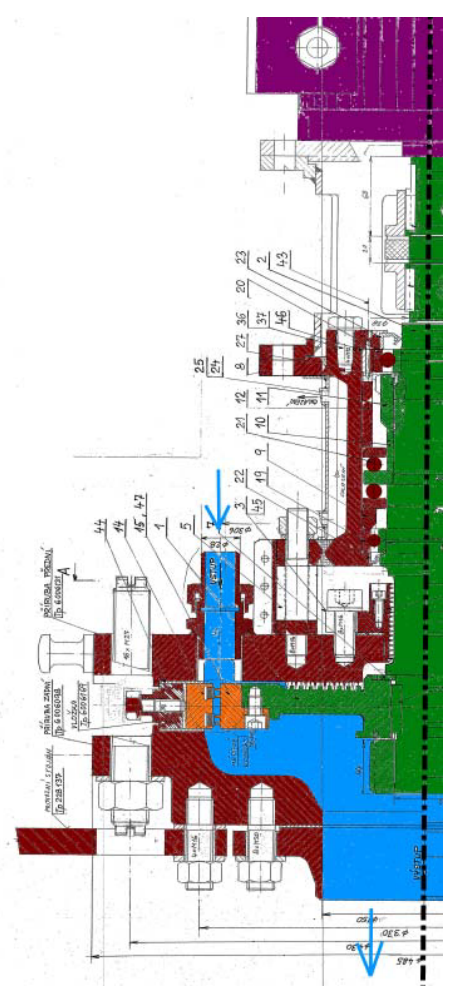

Fig. 4. Test rig section: motor(violet), shaft(green), stator(brown), test section(orange), steam flow-path(blue)

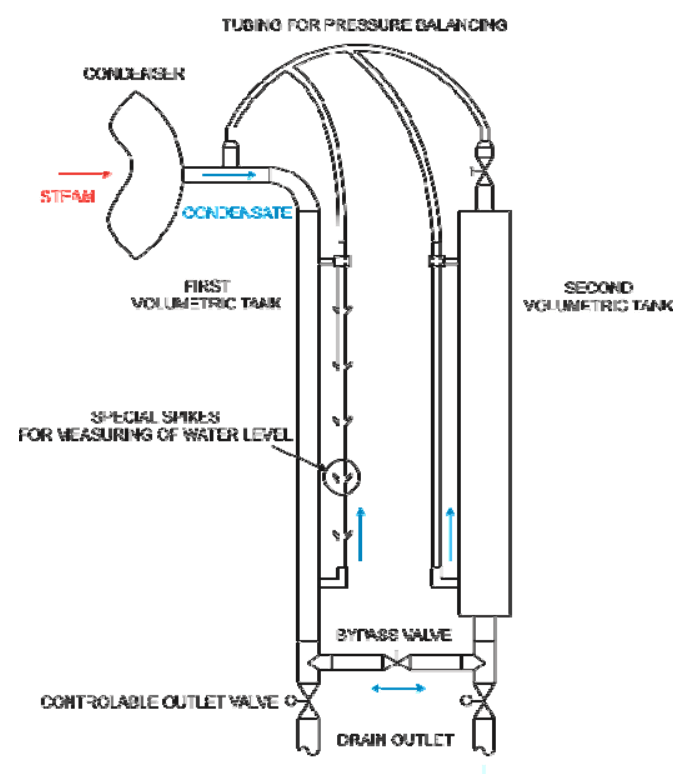

Fig. 5. Equipment for measuring the condensate volume (the leakage through the seal)

\section{Numerical calculations}

2D (SEAL) and 3D (FLUENT) calculation models were created. The models corresponded with real seals geometries which were tested by using the steam test rig described in previous chapter. An example of the 2D calculation model of a typical stepped labyrinth seal with four rotor teeth is shown on Figure 6.

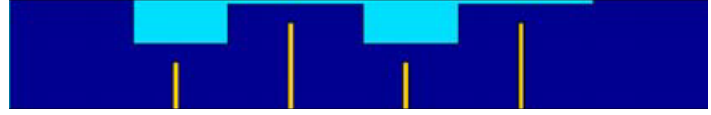

INLET

OUTLET

Fig. 6. 2D calculation model of a typical stepped labyrinth

The 3D calculation model was created as one quarter of the test rig included tested seal and measuring planes in the same location as real measuring pressure and temperature taps. An example of the model is shown on Figure 7.

For calculations carried out by FLUENT, the k- $\omega$ turbulence model for the steady compressible turbulent flow was used.

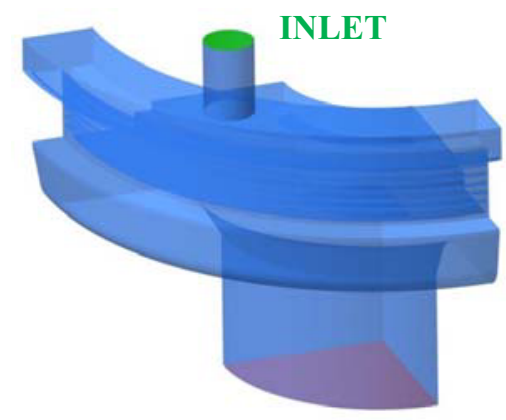

OUTLET

Fig. 7. 3D calculation model of one quarter of the test rig

\section{Results}

12 different seal geometries and clearances were tested, calculated and compared in a wide range of pressure ratios. For data summary, there were calculated average deviation values between experiment, analytical and numerical results.

The mass flow predicted by the Zalf formula is higher in case of the stepped as well as see-through labyrinth type. It is considerably more than the measured values. The experimental results are almost in agreement with the numerical calculations, which confirm that the Zalf formula really overestimates the seal leakage.

The results predicted by Samoylovich formula are significantly closer to the tested value with consideration the measurement uncertainty $\pm 5 \%$.

Table 1. Average deviations of the leakage from experiments

\begin{tabular}{|c|c|c|c|c|}
\hline Seal Type & $\begin{array}{c}\text { Zalf } \\
{[\%]}\end{array}$ & $\begin{array}{c}\text { Samoylovich } \\
{[\%]}\end{array}$ & $\begin{array}{c}\text { FLUENT } \\
{[\%]}\end{array}$ & $\begin{array}{c}\text { SEAL } \\
{[\%]}\end{array}$ \\
\hline Stepped & +46 & +5 & -12 & -6 \\
\hline See-through & +24 & +2 & +5 & +5 \\
\hline
\end{tabular}

The experimental results (Figure 8. and 9.) show some practical findings. For example, for leakage reduction, it is more effective to lower the radial clearance than to increase the number of the teeth.

Another finding is connected with the see-through seal. Using a higher number of the teeth, while reducing 
span, has a very limited effect. This often happens when trying to keep the overall size of the seal.

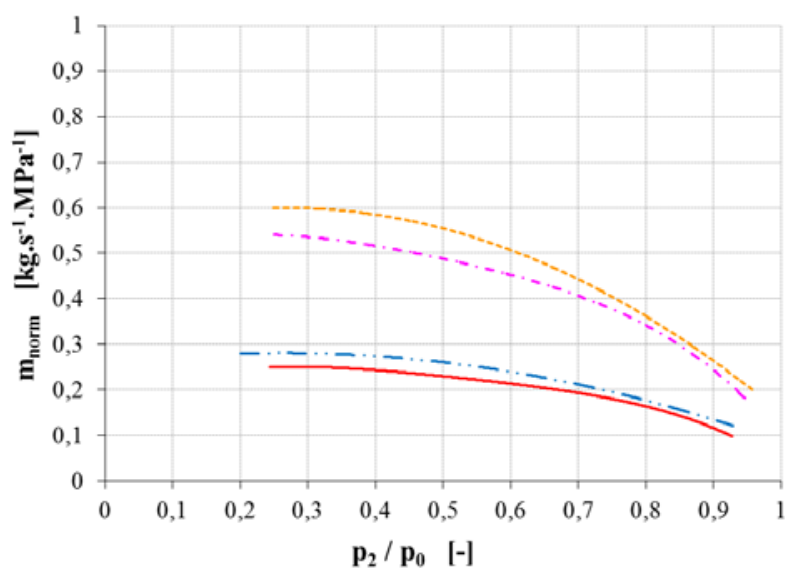

Stepped labyrinth with 3 teeth, clearance $0.38 \mathrm{~mm}$, teeth span $6.1 \mathrm{~mm}$ Stepped labyrinth with 3 teeth, clearance $0.90 \mathrm{~mm}$, teeth span $6.1 \mathrm{~mm}$

- Stepped labyrinth with 4 teeth, clearance $0.37 \mathrm{~mm}$, teeth span $6.0 \mathrm{~mm}$ Stepped labyrinth with 4 teeth, clearance $0.91 \mathrm{~mm}$, teeth span $6.0 \mathrm{~mm}$

Fig. 8. Experimental results for the stepped labyrinth seal

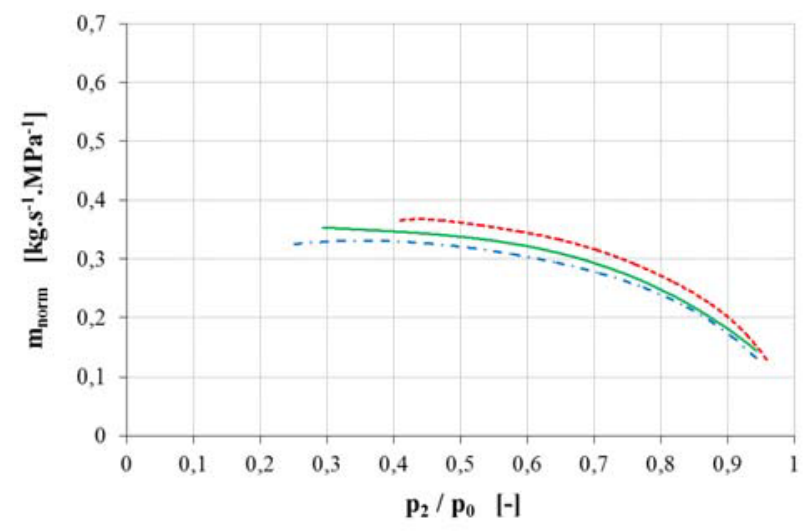

- See-through labyrinth with 2 teeth, clearance $0.41 \mathrm{~mm}$, teeth span $18.3 \mathrm{~mm}$ See-through labyrinth with 3 teeth, clearance $0.41 \mathrm{~mm}$, teeth span $9.0 \mathrm{~mm}$ See-through labyrinth with 6 teeth, clearance $0.40 \mathrm{~mm}$, teeth span $4.5 \mathrm{~mm}$

Fig. 9. Experimental results for the see-through labyrinth seal

\section{Conclusions}

The state of the art method (Zalf) for predicting seal leakage is limiting the achievable steam turbine efficiency. Therefore it was carried out the comparison between the experiments, analytical and numerical calculations. The experiments confirmed that the Zalf formula is too conservative and an alternative analytical formula has to be introduced to allow an improved turbine design.

The mass flow predicted by the Samoylovich empirical formula is very close to the experimental results. The approximate deviation $\pm 10 \%$ of the numerical data from the experimental and analytical data is sufficient in case of CFD calculations.

The new empirical formula for the see-through labyrinth seal correction coefficient was derived according to the results. Now, it is possible to better describe the flow behaviour in the see-through type of labyrinth seal by the new coefficient which ensures that the leakage prediction is more precise.

These conclusions have changed Doosan Skoda former analytical calculations of the seal losses. As a result, the leakage through the stepped and the seethrough labyrinth seals should be calculated by the Samoylovich empirical formula instead of the Zalf approach.

\section{References}

1. Aungier, R., H.: Turbine Aerodynamic: Axial-Flow and Radial-Inflow Turbine Design and Analysis. New York: ASME Press, 2006.

2. Stodola, A.: Dampf und Gas-Turbinen. Berlin, 1922. 3. Bednar, L.; Hoznedl, M.; Tajc, L.: Ucinnost turbinovych stupnu, III. upravene vydani. Vyzkumna zprava VZTP 1047, Plzen: Doosan Skoda Power, s.r.o., 2010.

4. Deych, M., E., Samoylovich, G., S.: Bases of Aerodynamics of Axial Turbines. Moscow, 1959.

5. Zalf, G., A., Zvyagintsev V., V.: Thermal calculation of Steam Turbines. Moscow, 1961.

6. Kearton, W., J.; Keh, T., H.: Leakage of Air through Labyrinth Glands of Staggered Type. University of Liverpool: 2013.

7. Shcheglyaev, A.V.: Steam Turbines. Moscow, 1967.

8. Egli, A.: The Leakage of Steam through Labyrinth Seals. Transactions ASME Trans, vol. 57, pp. 115-122.

9. Kearton W. J.: The Flow of Air through Radial Labyrinth Glands. Proceedings of the Institution of Mechanical Engineers, Vol. 169, 1955.

10. Trutnovsky, K.: Contactless Seals, Foundations and Applications of Flows through Slots and Labyrinths. NASA TT F 17, 352, April 1977.

11. Yoon, S.: The Effect of the Degree of Reaction on the Leakage Loss in Steam Turbines. ASME Turbo Expo, GT2012-69602, Copenhagen, June 2012. 